\title{
ComunicaTEC: Tecnologias de Comunicação para Educação e Colaboração
}

\author{
Mariano Pimentel \\ Departamento de Informática Aplicada - CCET - UNIRIO \\ Av. Pasteur, 458, sala 114 - cep: 22290-240, Urca, Rio de Janeiro, RJ, Brasil \\ pimentel@uniriotec.br
}

\begin{abstract}
Resumo. Este artigo apresenta o projeto ComunicaTEC cujo objetivo é investigar o uso e promover o desenvolvimento de ferramentas de comunicação voltadas para a Educação e o Trabalho em grupo. Este projeto adota o Modelo de Trabalho para análise e projeto de ferramentas para colaboração. É apresentado um estudo de caso com uma ferramenta para a realização de entrevistas. O estudo de caso exemplifica a aplicação dos procedimentos de pesquisa e desenvolvimento elaborados neste projeto.
\end{abstract}

\section{Introdução}

O projeto ComunicaTEC investiga o uso e o desenvolvimento de tecnologias de comunicação para Educação e Colaboração. Por um lado, uma ferramenta computacional impõe restrições para a aplicação de uma técnica de trabalho em grupo, sugerindo modificações da técnica para torná-la mais adequada à ferramenta em uso. Por outro lado, uma técnica apresenta peculiaridades que sugerem modificações na ferramenta para torná-la mais adequada à técnica. O projeto ComunicaTEC reconhece esta influência mútua e investiga concomitantemente o desenvolvimento de ferramentas de comunicação e seu uso para aplicação de técnicas de trabalho em grupo.

O computador, originalmente voltado para o processamento de dados, tornou-se um meio de comunicação. Correio-eletrônico (email), fórum, lista de discussão, blog, bate-papo (chat), videoconferência, mensagem-instantânea (messenger) e VoIP (comunicação de voz pela Internet) são algumas das ferramentas computacionais de comunicação mais conhecidas (Fuks et al., 2003). Estas ferramentas foram desenvolvidas para transmitir uma mensagem genérica sem considerar as peculiaridades da atividade que será realizada através da ferramenta. É preciso adequar as ferramentas de comunicação para a aplicação de técnicas específicas.

$\mathrm{Na}$ atual sociedade, que se transforma da industrial para a conectada (ou da informação), cada vez mais o sujeito deixa de trabalhar individualmente para trabalhar em grupo. Dentre as técnicas mais conhecidas de trabalho em grupo, destacam-se: debate, entrevista, tempestade de idéias (brainstorming), fórum, mesa-redonda, painel, júri-pedagógico ou simulado, desempenho de papéis (roleplaying), problem-solvinggroup, workshop, conferência, seminário etc [Minicucci, 2001]. Estas técnicas já são usadas para educação e trabalho sem, contudo, terem sido elaboradas para a realização através do computador. É preciso adequar estas técnicas para que sejam aplicadas pelo computador, objetivando tirar vantagens do potencial e adequar-se às limitações deste meio. 
É preciso alinhar a pesquisa destas duas áreas - adequar as ferramentas computacionais para a aplicação de técnicas específicas de trabalho em grupo, bem como adequar as técnicas para que sejam mediadas pelo computador. Neste projeto de pesquisa, investiga-se os problemas que ocorrem com o uso da tecnologia na aplicação da técnica. Visando resolver os problemas identificados, desenvolve-se uma nova versão da ferramenta ou, ocasionalmente, uma adequação da técnica. Um novo estudo de caso é executado para realimentar a pesquisa e o desenvolvimento. O objetivo é identificar quais são os mecanismos para elaborar boas ferramentas para a aplicação de uma técnica específica de trabalho em grupo.

\section{Trabalho em Grupo}

Neste projeto de pesquisa, o trabalho é analisado em função do Modelo de Trabalho, baseado na Teoria da Atividade (Nardi, 2002), apresentado na Figura 1. Segundo este modelo, o trabalho é a transformação de um objeto num produto pela atuação de um grupo seguindo uma técnica e usando uma ferramenta.

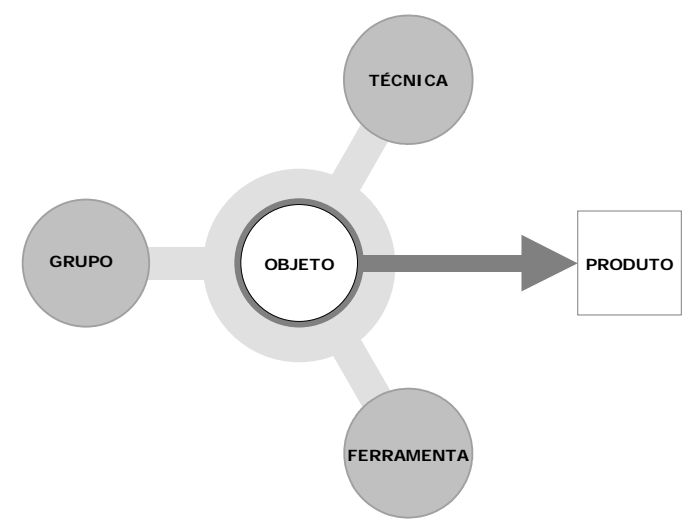

Figura 1. Modelo de Trabalho

Todos, de alguma forma, trabalham em grupo; ninguém trabalha completamente sozinho, independentemente de outros. Numa indústria, os vários funcionários trabalham juntos, ainda que cada funcionário execute uma atividade altamente especializada e não conheça completamente a técnica para gerar o produto final. Na prestação de serviço, o cliente trabalha com o profissional para que seja gerado o produto esperado.

Uma técnica de trabalho especifica as atividades que devem ser executadas na geração do produto. Por exemplo, para elicitar os requisitos de um software, em reuniões entre membros do projeto e interessados (clientes e usuários), são sugeridas as técnicas de brainstorming, entrevista, workshop, storyboarding, e roleplaying [Kruchten, 1999]. Para a educação online, são sugeridas técnicas como seminário, debate e pequenos grupos de trabalho [Lucena et al., 2006].

Ferramentas são os instrumentos usados para realizar ou auxiliar a transformação de um objeto inicial no produto final do trabalho. Duas ferramentas diferentes podem ser usadas para executar uma mesma técnica; uma única ferramenta pode ser usada em diferentes técnicas de trabalho. 
No projeto de pesquisa aqui apresentado: o grupo é caracterizado em função dos Papéis: habilidades e responsabilidades esperadas de um indivíduo ou equipe; a técnica é caracterizada pela seqüência de atividades na elaboração dos artefatos intermediários até o produto final, representada como um diagrama de atividade da UML onde também se representa o mapeamento papéis-atividades-artefatos; e a ferramenta é caracterizada pelas funcionalidades e mecanismos usados para a realização das atividades. O estudo de caso apresentado na próxima seção exemplifica o uso destes procedimentos para o desenvolvimento de uma ferramenta computacional para entrevistas.

Sabe-se que a aplicação de algumas técnicas de trabalho em grupo pode até gerar resultados melhores quando mediadas por computador do que quando realizadas presencialmente, como evidenciado com a aplicação da técnica brainstorming [Gallupe et al., 1992]. Às vezes é preciso modificar a técnica para obter vantagens do potencial e adequar-se às limitações da ferramenta computacional - como exemplificado com a modificação da técnica de debate para aproveitar-se das técnicas de conversação desenvolvidas para o Mediated Chat 2.0 [Rezende, 2003], e exemplificado com a modificação da técnica de seminário, adotando o modelo IBIS de argumentação, para tirar proveito da estruturação em árvore disponível no serviço Conferências do AulaNet [Gerosa et al., 2004].

Além da adequação das técnicas de trabalho em grupo, o projeto ComunicaTEC volta-se para a adequação das ferramentas computacionais para a aplicação de tais técnicas. Para realizar este tipo de pesquisa, mantém-se inalterados o grupo e a técnica, e varia-se a ferramenta. Dados para inferir as influências da variação da ferramenta são: os artefatos produzidos (ex.: registro das mensagens trocadas, analisado a partir de técnicas de análise do discurso), dados coletados do processo usado na realização das atividades, e declarações dos usuários obtidas com formulários ou entrevistas de perguntas abertas [Nicolaci-da-Costa et al., 2001]. Com estes procedimentos, procura-se inferir se a nova ferramenta possibilita a realização de determinadas atividades que antes não tinham suporte computacional, ou se melhorou a realização das atividades em comparação com a versão anterior da ferramenta [Pimentel et al., 2006; Fuks et al., 2006].

\section{Estudo de caso: ferramenta "Entreviste" para educação}

Em artigos anteriores, foram apresentadas pesquisas de como a ferramenta de bate-papo tem sido modificada para ser usada na realização de debates educacionais [Fuks et al., 2006a, 2006b]. Nesta seção, discute-se como a ferramenta de bate-papo também pode ser modificada para ser usada na realização de entrevistas. Este estudo de caso exemplifica como o corpo teórico do projeto ComunicaTEC é colocado em prática no desenvolvimento de sistemas colaborativos. Discute-se como caracterizar o grupo, a técnica e a ferramenta; e discute-se o processo de desenvolvimento da ferramenta visando adequá-la para a realização de uma técnica específica.

\subsection{Grupo}

A realização de entrevistas envolve os papéis identificados e apresentados no Quadro 1 (Fávero, 2000; Minicucci, 2001; Lage, 2003). 


\section{Quadro 1. Papéis para a realização de Entrevista}

\begin{tabular}{|c|c|}
\hline Papel & Habilidades e Responsabilidades \\
\hline $\begin{array}{l}\text { Entrevistado } \\
\text { (Especialista) }\end{array}$ & $\begin{array}{l}\text { Atua neste papel quem possui informações de interesse e tem a responsabilidade de } \\
\text { transmiti-las respondendo a perguntas dos entrevistadores. No contexto educacional, } \\
\text { este papel deve ser exercido por um convidado ou pelo próprio professor. }\end{array}$ \\
\hline Entrevistador & $\begin{array}{l}\text { Pessoa com a responsabilidade de obter informações do entrevistado. Deve possui } \\
\text { domínio da técnica de interrogar: propor perguntas adequadas e reformular o } \\
\text { questionamento em função das respostas. No contexto educacional, este papel deve } \\
\text { ser exercido por um grupo selecionado de alunos ou mesmo por todos os alunos da } \\
\text { turma. }\end{array}$ \\
\hline Moderador & $\begin{array}{l}\text { Tem a responsabilidade de manter a ordem e o andamento da entrevista. Em casos de } \\
\text { entrevista moderada, este papel também é responsável por selecionar as perguntas dos } \\
\text { entrevistadores. No contexto educacional, este papel deve ser exercido pelo professor. }\end{array}$ \\
\hline Espectador & $\begin{array}{l}\text { Tem interesse nas informações do entrevistado, mas não tem a função de interrogá-lo } \\
\text { (exceção pode ser feita no final da entrevista). No contexto educacional, este papel } \\
\text { pode ser desempenhado pela maioria dos alunos da turma, ou então não ser } \\
\text { desempenhado no caso em que todos os alunos atuam como entrevistador. }\end{array}$ \\
\hline Sintetizador & $\begin{array}{l}\text { Tem a função de elaborar uma síntese da entrevista. No contexto educacional, pode } \\
\text { ser desempenhado por } 2 \text { ou } 3 \text { alunos da turma, ou pelo próprio professor. }\end{array}$ \\
\hline
\end{tabular}

Para a realização de uma entrevista, os participantes devem ser organizados para exercer os papéis apresentados no Quadro 1. No quadro, também foi sugerida a organização dos participantes de uma turma para a realização de entrevistas educacionais.

\subsection{Dinâmica}

Neste estudo de caso, a técnica investigada foi a de entrevista não-moderada, cujo diagrama de atividades é apresentado na Figura 2. Nesta técnica, o entrevistador faz perguntas diretamente para o entrevistado sem mediação do moderador.

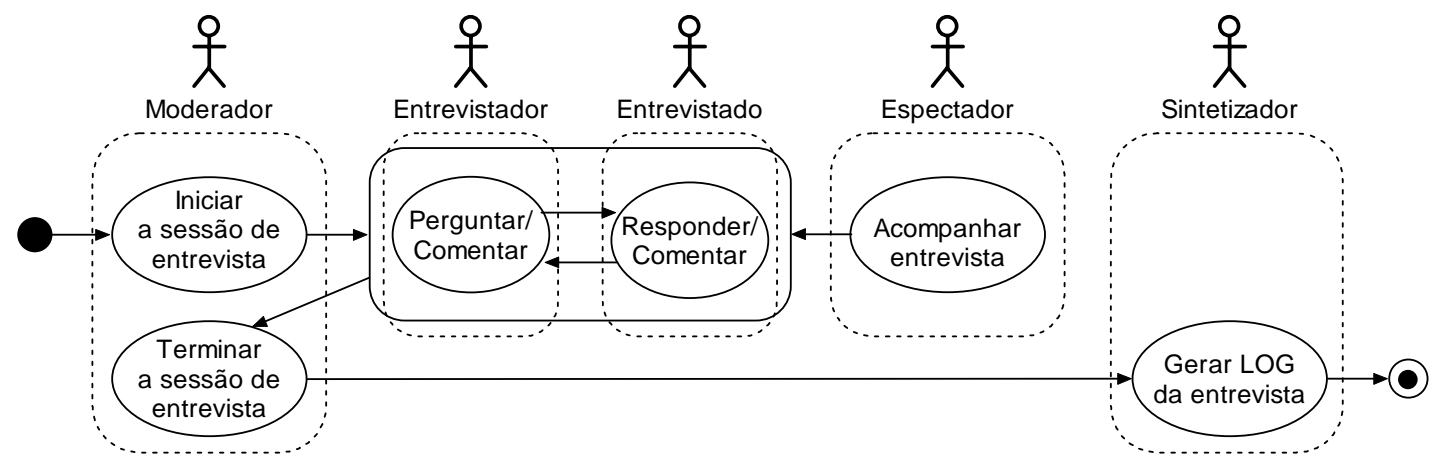

Figura 2. Técnica: Entrevista não-moderada

A técnica de trabalho em grupo, formalizada através de um Diagrama de Atividades como o ilustrado na Figura 2, torna-se útil para: auxiliar análises visando possíveis modificações da técnica; ou auxiliar a especificação de um novo software para dar suporte àquele fluxo de trabalho (auxilia, por exemplo, na elaboração do diagrama de casos de uso); ou mesmo para auxiliar a análise da ferramenta em uso (a ferramenta computacional está dando suporte adequado a uma determinada atividade? será que há alguma atividade que deveria ter um suporte computacional?). 


\subsection{Ferramenta}

Com o objetivo de desenvolver uma nova ferramenta, é importante caracterizar a família de aplicações do domínio. Em relação às ferramentas para entrevistas, foram analisadas as instâncias disponíveis nos principais portais brasileiros: UOL, Terra, IG e Globo (de acordo com o índice de audiência, Ibope Nielsen/NetRating, 2006).

Quadro 2. Análise das ferramentas de entrevista de portais brasileiros

\begin{tabular}{|c|c|c|c|c|c|}
\hline \multicolumn{2}{|c|}{$\begin{array}{l}\text { Elementos } 3 \mathrm{C} \\
\text { para análise }\end{array}$} & \multirow{2}{*}{$\begin{array}{l}\begin{array}{l}\text { UOL Batepapo } \\
\text { com convidados }\end{array} \\
\begin{array}{l}\text { texto de todos; vídeo } \\
\text { só do entrevistado }\end{array}\end{array}$} & \multirow{2}{*}{$\begin{array}{l}\begin{array}{l}\text { Terra Chat } \\
\text { Entrevistas }\end{array} \\
\text { texto e vídeo do } \\
\text { entrevistado }\end{array}$} & \multirow{2}{*}{$\begin{array}{l}\text { I G } \\
\text { Papo Agenda } \\
\text { texto }\end{array}$} & \multirow{2}{*}{$\begin{array}{l}\text { Globo } \\
\text { VideoChat } \\
\text { texto e áudio só do } \\
\text { entrevistado e do } \\
\text { moderador }\end{array}$} \\
\hline 电 & Linguagem & & & & \\
\hline$\frac{U}{2}$ & Transmissão & $\begin{array}{l}\text { texto pontual, vídeo } \\
\text { contínuo }\end{array}$ & texto pontual & texto pontual & $\begin{array}{l}\text { texto pontual, áudio } \\
\text { contínuo }\end{array}$ \\
\hline ํํㅇ & $\begin{array}{l}\text { Estruturação do } \\
\text { discurso }\end{array}$ & linear & linear & linear & linear \\
\hline & Categorização & tipo de fala & tipo de fala & tipo de fala & sem categorização \\
\hline \multirow{8}{*}{ 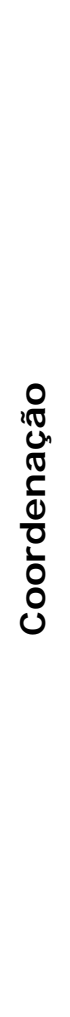 } & Tópico & $\operatorname{sim}$ & $\operatorname{sim}$ & $\operatorname{sim}$ & $\operatorname{sim}$ \\
\hline & Sessão & $\operatorname{sim}$ & $\operatorname{sim}$ & $\operatorname{sim}$ & $\operatorname{sim}$ \\
\hline & Presença & $\begin{array}{l}\text { entrevistado, e } \\
\text { entrevistadores na } \\
\text { mesma sala (não lista } \\
\text { moderador) }\end{array}$ & $\begin{array}{l}\text { entrevistadores na } \\
\text { sala (não lista } \\
\text { moderadores nem } \\
\text { entrevistados) }\end{array}$ & $\begin{array}{l}\text { entrevistado e } \\
\text { entrevistadores na } \\
\text { mesma sala }\end{array}$ & $\begin{array}{l}\text { entrevistadores(não } \\
\text { lista moderadores nem } \\
\text { entrevistados) }\end{array}$ \\
\hline & Disponibilidade & estado único: online & estado único: online & estado único: online & estado único: online \\
\hline & Posse da palavra & moderada & moderada & moderada & moderada \\
\hline & Freqüência & $\begin{array}{l}\text { para o entrevistado, } \\
\text { mensagens limitada } \\
\text { pelo moderador; } \\
\text { irrestrito para os } \\
\text { demais na sala }\end{array}$ & $\begin{array}{l}\text { para o entrevistado, } \\
\text { mensagens limitada } \\
\text { pelo moderador; } \\
\text { irrestrito para os } \\
\text { demais na sala }\end{array}$ & $\begin{array}{l}\text { para o entrevistado, } \\
\text { mensagens limitada } \\
\text { pelo moderador; } \\
\text { irrestrito para os } \\
\text { demais na sala }\end{array}$ & $\begin{array}{l}\text { sempre limitada pelo } \\
\text { moderador }\end{array}$ \\
\hline & $\begin{array}{l}\text { Turno-em- } \\
\text { desenvolvimento }\end{array}$ & sem indicações & sem indicações & sem indicações & $\begin{array}{l}\text { barulho da digitação } \\
\text { junto com o áudio do } \\
\text { entrevistado }\end{array}$ \\
\hline & Avaliação & não & não & não & não \\
\hline \multirow{3}{*}{$\begin{array}{l}0 \\
\text { Ư } \\
0 \\
0 \\
0 \\
0 \\
0 \\
0 \\
0\end{array}$} & Registro & $\begin{array}{l}\text { repositório editado de } \\
\text { entrevistas anteriores; } \\
\text { registra: hora, } \\
\text { emissor, destinatário, } \\
\text { categoria e conteúdo } \\
\text { da mensagem }\end{array}$ & $\begin{array}{l}\text { repositório editado de } \\
\text { entrevistas anteriores; } \\
\text { registra: hora, } \\
\text { emissor, destinatário, } \\
\text { categoria e conteúdo } \\
\text { da msg; }\end{array}$ & $\begin{array}{l}\text { registra: hora, } \\
\text { emissor, destinatário, } \\
\text { categoria e conteúdo } \\
\text { da mensagem }\end{array}$ & $\begin{array}{l}\text { repositório editado de } \\
\text { entrevistas anteriores; } \\
\text { registra: emissor e } \\
\text { conteúdo da } \\
\text { mensagem }\end{array}$ \\
\hline & $\begin{array}{l}\text { Configuração do } \\
\text { espaço }\end{array}$ & $\begin{array}{l}\text { janela única, com } \\
\text { destaque para as } \\
\text { mensagens de } \\
\text { mediador e do } \\
\text { entrevistado }\end{array}$ & $\begin{array}{l}\text { janela única, com } \\
\text { destaque para as } \\
\text { mensagens de } \\
\text { mediador e do } \\
\text { entrevistado }\end{array}$ & $\begin{array}{l}\text { janela única, com } \\
\text { destaque para as } \\
\text { mensagens de } \\
\text { mediador e do } \\
\text { entrevistado }\end{array}$ & $\begin{array}{l}\text { janela única, com } \\
\text { destaque para as } \\
\text { mensagens de } \\
\text { mediador e do } \\
\text { entrevistado }\end{array}$ \\
\hline & $\begin{array}{l}\text { Mensagens } \\
\text { preconcebidas }\end{array}$ & não & não & não & não \\
\hline
\end{tabular}

Anais do III Simpósio Brasileiro de Sistemas de Informação. Curitiba, PR, novembro de 2006. 
Para realizar a análise das ferramentas de entrevista, foi usado o Framework Conceitual 3C do domínio Ferramentas de Comunicação Síncrona (Pimentel et al., 2006). O resultado desta análise é apresentado no Quadro 2.

A partir do uso destas ferramentas para a realização de entrevistas, foram identificados os seguintes problemas:

- o texto do entrevistado se mistura com o dos entrevistadores, sem uma clara separação do diálogo;

- é difícil compreender, às vezes, a qual pergunta uma resposta se refere;

- com os vários entrevistadores, e sem um moderador, o entrevistado é abordado com um número excessivo de perguntas;

- sem um vídeo, a audiência fica desconfiada da identidade do entrevistado;

- quando o entrevistado digita frases longas, a audiência sente como se ele estivesse demorando a responder.

As ferramentas analisadas não lidam adequadamente com a principal característica de uma entrevista: a organização dos pares conversacionais perguntaresposta, potencializando a ocorrência dos dois primeiros problemas listados. A ferramenta "Entreviste" (Pessoa, 2002), Figura 3, exemplifica como poderia ser uma ferramenta mais específica para a realização de entrevistas. Na ferramenta Entreviste, cada resposta do entrevistado é precedida pela pergunta do entrevistador - o que evidencia o par dialógico pergunta-resposta. As mensagens são organizadas em duas áreas distintas: perguntas dos entrevistadores (geralmente vários), e respostas do entrevistado (geralmente único) - o que enfatiza a assimetria do diálogo.

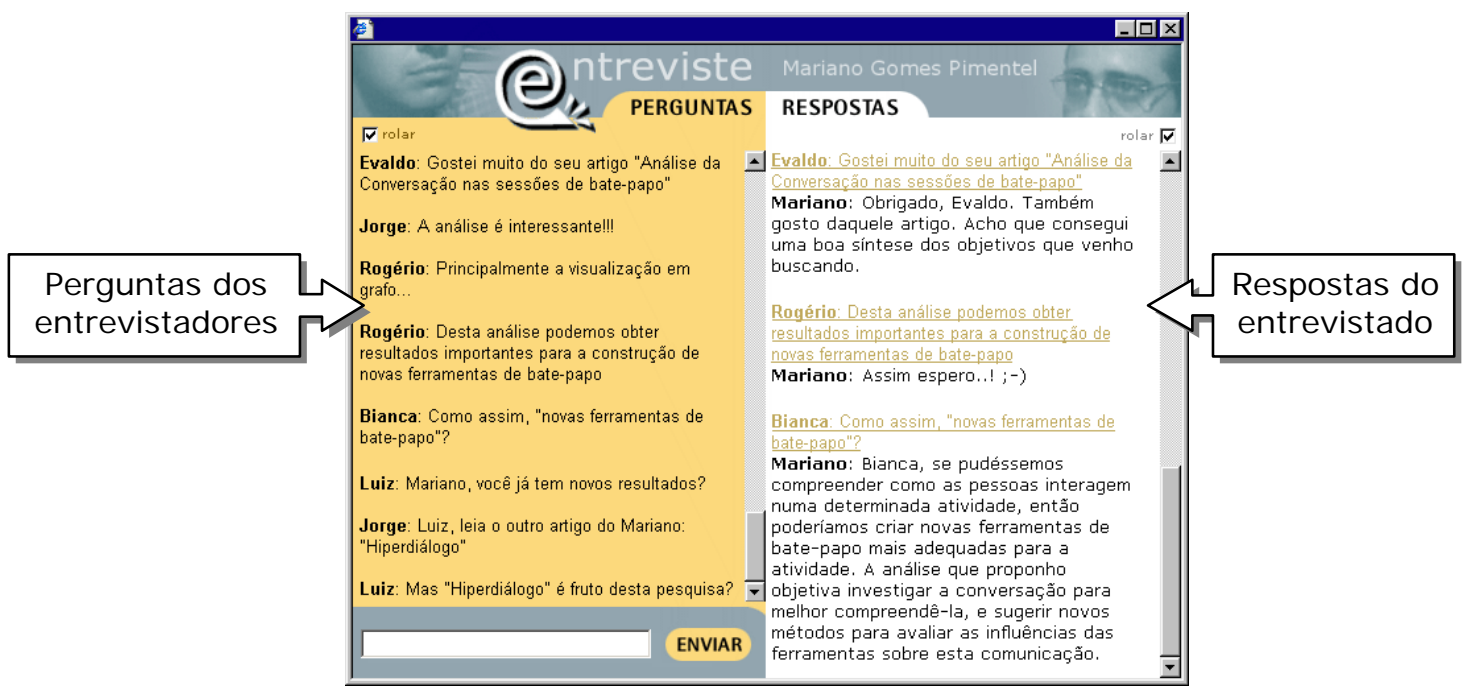

Figura 3. Ferramenta Entreviste

A hipótese é que a ferramenta Entreviste, em comparação com as demais ferramentas analisadas, diminui problemas relacionados à dificuldade de identificação do par dialógico pergunta-resposta. A partir de um primeiro estudo de caso realizado com a ferramenta Entreviste (Pessoa, 2000), os usuários mostraram-se satisfeito com a ferramenta e declararam, durante a realização da própria atividade, terem conseguido acompanhar e compreender melhor o diálogo. 
Esta primeira versão da ferramenta Entreviste foi desenvolvida para resolver dois dos problemas identificados com o uso das ferramentas semelhantes. Em trabalhos futuros, espera-se melhorar esta versão visando resolver os demais problemas já identificados. Também objetiva-se realizar novos estudos de caso com o uso desta ferramenta na realização de entrevistas no contexto educacional.

\section{Conclusão e trabalho futuro}

Este artigo apresentou o projeto de pesquisa ComunicaTEC, que investiga o uso e o desenvolvimento de tecnologias de comunicação para Educação e Colaboração. Neste projeto é feito uso do Modelo de Trabalho para guiar a análise e projeto das ferramentas. A partir deste modelo, procura-se caracterizar o grupo, a técnica e a ferramenta usada no trabalho colaborativo. Um estudo de caso, sobre o desenvolvimento de uma ferramenta de entrevista, ilustrou a aplicação deste modelo para a pesquisa e o desenvolvimento das ferramentas de comunicação.

Sucessivas versões da ferramenta Entreviste ainda serão desenvolvidas visando torná-la ainda mais adequada para a realização de entrevistas. Com o estudo de caso já realizado, conclui-se que os procedimentos elaborados no contexto do projeto ComunicaTEC são úteis para a pesquisa e o desenvolvimento de ferramentas para colaboração.

\section{Referências}

FÁVERO, L.L. (2000) A entrevista na fala e na escrita. Fala e escrita em questão. Dino Preti (org). São Paulo: Humanistas/FFLCH/USP.

FUKS, H., GEROSA, M.A. \& PIMENTEL, M. (2003) Projeto de Comunicação em Groupware: Desenvolvimento, Interface e Utilização. XXII Jornada de Atualização em Informática, Anais do XXIII Congresso da Sociedade Brasileira de Computação, v2, cap. 7, p. 295-338.

FUKS, H., PIMENTEL, M., GEROSA, M.A., FERNANDES, M.C.P., LUCENA, C.J.P. (2006a) Novas estratégias de avaliação online: aplicações e implicações em um curso totalmente a distância através do ambiente AulaNet. In: Avaliação da Aprendizagem em Educação Online, M. SILVA e E. SANTOS (orgs.), São Paulo: Loyola. p. 369-385.

FUKS, H., PIMENTEL, M., LUCENA, C.J.P. (2006b) R-U-Typing-2-Me? Evolving a chat tool to increase understanding in learning activities. International Journal of Computer-Supported Collaborative Learning, v. 1. Springer. p. 117-142.

GALLUPE, R.B., DENNIS, A.R., COOPER, W.H., VALACICH, J.S., BASTIANUTTI, L.M., NUNAMAKER, J.F. (1992) Eletronic Brainstorming and group size. Academy of Management Journal, v. 35, n. 2, p. 350-369.

GEROSA, M. A., FUKS, H., LUCENA, C.J.P. (2004) Estruturação e Categorização de Mensagens em Ferramentas de Comunicação Textuais Assíncronas. In: Electronic Proceedings of the World Congress on Engineering and Technology Education, WCETE'2004. Santos - SP.

KRUCHTEN, P. (1999) The Rational Unified Process - an introduction. Addison-Wesley-Longman, Reading, MA.

LAGE, N. (2003) A reportagem - teoria e técnica de entrevista, 3 ed. Record.

LUCENA, C.J.P., FUKS, H., RAPOSO, A., GEROSA, M.A., PIMENTEL, M. (2006) Communication, Coordination and Cooperation in Computer-Supported Learning: The AulaNet Experience. in: Advances in Computer-Supported Learning, F.M.M. Neto and F. Brasileiro (orgs), p. 274-297.

MINICUCCI, A. (2001) Técnicas do trabalho de grupo, 3. ed. São Paulo : Atlas. 
NARDI, B.A., REDMILE, D. (eds) (2002) Activity Theory and the practice of Design. CSCW 2002 Computer Supported Cooperative Work, v. 11, v. 1-2.

NICOLACI-DA-COSTA, A. M., LEITÃO, C. F. e ROMÃO-DIAS, D. (2001) Gerando conhecimento sobre homens, mulheres e crianças que usam computadores: algumas contribuições da psicologia clínica, IV Workshop sobre Fatores Humanos em Sistemas Computacionais, Florianópolis.

PIMENTEL, M., GEROSA, M.A., FILIPPO,D., RAPOSO, A., FUKS, H., LUCENA, C.J.P. (2006) Modelo 3C de Colaboração no desenvolvimento de sistemas colaborativos. Anais Simpósio Brasileiro de Sistemas Colaborativos. Natal - RN, Novembro 2006.

REZENDE, J.L. (2003) Aplicando Técnicas de Comunicação para a Facilitação de Debates no Ambiente AulaNet. Dissertação de Mestrado, Departamento de Informática, Pontifícia Universidade Católica do Rio de Janeiro (PUC-Rio). 